\title{
Structure and Spectroscopy of Ground and Excited States of LiYb
}

\section{Citation}

Zhang, Peng, Hossein R. Sadeghpour, and Alexander Dalgarno. 2010. Structure and spectroscopy of ground and excited states of LiYb. Journal of Chemical Physics 133(4): 044306.

\section{Published Version}

doi:10.1063/1.3462245

\section{Permanent link}

http://nrs.harvard.edu/urn-3:HUL.InstRepos:5132921

\section{Terms of Use}

This article was downloaded from Harvard University's DASH repository, and is made available under the terms and conditions applicable to Open Access Policy Articles, as set forth at http:// nrs.harvard.edu/urn-3:HUL.InstRepos:dash.current.terms-of-use\#OAP

\section{Share Your Story}

The Harvard community has made this article openly available.

Please share how this access benefits you. Submit a story.

Accessibility 


\title{
Structure and spectroscopy of ground and excited states of LiYb
}

\author{
P. Zhang, H. R. Sadeghpour, and A. Dalgarno ${ }^{\text {a) }}$ \\ ITAMP, Harvard-Smithsonian Center for Astrophysics, 60 Garden St., \\ Cambridge, Massachusetts 02138, USA
}

(Received 6 April 2010; accepted 18 June 2010; published online 28 July 2010)

\begin{abstract}
Multireference configuration interaction and coupled cluster calculations have been carried out to determine the potential energy curves for the ground and low-lying excited states of the LiYb molecule. The scalar relativistic effects have been included by means of the Douglas-Kroll Hamiltonian and effective core potential and the spin-orbit couplings have been evaluated by the full microscopic Breit-Pauli operator. The LiYb permanent dipole moment, static dipole polarizability, and Franck-Condon factors have been determined. Perturbations of the vibrational spectrum due to nonadiabatic interactions are discussed. (c) 2010 American Institute of Physics.
\end{abstract}

[doi:10.1063/1.3462245]

\section{INTRODUCTION}

The production of cold and ultracold atomic ensembles has greatly expanded the traditional domain of atomic physics and created a much broader interdisciplinary field. The impact of creating ultracold molecules is expected to be just as profound as that of ultracold atoms. ${ }^{1}$ Molecules offer microscopic degrees of freedom absent in atomic gases. Polar molecules are of particular interest due to the existence of a permanent dipole, responsible for dipolar interaction among the molecules. This interaction is long-range and anisotropic. Many applications of polar molecules have been proposed. ${ }^{2}$ The Bardeen-Cooper-Schrieffer, Bose-Einstein condensation cross over has been investigated by creating Cooper pairs and Bose condensed Feshbach molecule of ${ }^{40} \mathrm{~K}$ atoms. ${ }^{3}$ Polar molecules $\mathrm{KRb}$ (Ref. 4) and RbCs (Ref. 5) in the ground state have been produced. Polar molecules may provide a promising platform for quantum information ${ }^{6}$ and lead to fundamentally new condensed-matter phases and complex quantum dynamics. ${ }^{7}$ The formation of an ensemble of ultracold molecules can facilitate ultracold chemistry and controlled chemical reaction. ${ }^{8}$

Experimental production of ultracold molecules is challenging. Techniques include buffer gas cooling, ${ }^{9}$ Stark deceleration of polar molecules, magnetically tunable Feshbach resonances, ${ }^{10}$ and light-assisted photoassociation. ${ }^{11}$ Ensemble mixtures of ultracold atoms is promising for the production of translationally cold molecules. The most recent demonstration of the formation of ultracold alkali-metal and Yb-containing molecule is RbYb. ${ }^{12}$

The rare-earth ytterbium $(\mathrm{Yb})$ is unique. Its electronic structure resembles that of the alkaline-earth atoms and it has seven stable isotopes: five bosons and two fermions. The trapping and cooling of the $\mathrm{Yb}$ atomic gas have been achieved $^{13}$ and the study of Yb-containing heteronuclear molecules with alkali-metal atoms is promising. The combination of alkali-metal and $\mathrm{Yb}$ atoms have permanent electric

\footnotetext{
${ }^{\text {a) }}$ Author to whom correspondence should be addressed. Electronic mail: adalgarno@cfa.harvard.edu.
}

dipole moments. Among the possible combinations, the $\mathrm{LiYb}$ molecule stands out for its versatility in the number of isotopic combinations allowing for the study of various molecular mixture species. Furthermore, ${ }^{6} \mathrm{Li}^{174} \mathrm{Yb}$ has the largest mass ratio ( $\sim 29)$ between $\mathrm{Yb}$ and alkali metal elements. The weakly bound $\mathrm{LiYb}$ molecules are expected to be collisionally stable and promising for the study of mass-imbalanced three-body systems such as Efimov trimer states. ${ }^{14}$ Experimental production of cold LiYb is in progress ${ }^{15}$ and theoretical input is needed.

The molecular interaction potentials, transition frequencies, radial derivative couplings, and spin-orbit couplings are necessary for understanding the mechanisms for production, collisional cooling, and relaxation of molecules. In this work, we present structure calculations of potential energy curves (PECs) of the ground and low-lying excited states of the $\mathrm{LiYb}$ molecule, the long-range van der Waals interaction coefficients, transition frequencies, static dipole polarizabilities, and permanent electric dipole moment of the molecule. We systematically explore effects due to electron correlation, basis set superposition error, and relativistic corrections.

\section{COMPUTATIONAL DETAILS}

For brevity of notations, the ground state asymptote of the $\mathrm{LiYb}$ molecule $\left[\mathrm{Li}\left({ }^{2} \mathrm{~S}\right)+\mathrm{Yb}\left({ }^{1} \mathrm{~S}\right)\right]$ will be denoted as $\mathrm{M}_{\mathrm{I}}$ and the second and third molecular asymptotes $\left[\mathrm{Li}\left({ }^{2} \mathrm{P}\right)\right.$ $\left.+\mathrm{Yb}\left({ }^{1} \mathrm{~S}\right)\right]$ and $\left[\mathrm{Li}\left({ }^{2} \mathrm{~S}\right)+\mathrm{Yb}\left({ }^{3} \mathrm{P}\right)\right]$ as $\mathrm{M}_{\mathrm{II}}$ and $\mathrm{M}_{\mathrm{III}}$.

The molecular properties of systems containing heavy elements such as $\mathrm{Yb}$ are significantly influenced by electron correlation and relativistic effects. For the LiYb molecule, scalar relativistic effects are dominant for the ground state. For the states associated with $\mathrm{M}_{\mathrm{II}}$, the spin-orbit coupling (SOC) with states from $\mathrm{M}_{\text {III }}$ could be important because of the relatively small asymptotic energy separation of $\sim 0.3 \mathrm{eV}$ and the relatively large SOC of $\mathrm{Yb}\left({ }^{3} \mathrm{P}\right)$. The fourth asymptote of $\mathrm{LiYb}$ correlates to $\mathrm{Li}\left({ }^{2} \mathrm{~S}\right)$ and an excited state of $\mathrm{Yb}$ resulting from the $4 f-5 d$ transition. Asymptotically, it is about $0.5 \mathrm{eV}$ above $\mathrm{M}_{\mathrm{III}}$ and thus will have little effect on 
the molecular states associated with $\mathrm{M}_{\mathrm{II}}$. We did not include this asymptote in our calculations. For states associated with $\mathrm{M}_{\mathrm{III}}$, it may have large effects.

\section{A. Relativistic corrections}

In our previous studies of the electric and optical fields response properties of the $\mathrm{Yb}$ atom, ${ }^{16}$ we found that the scalar relativistic effects could be satisfactorily approximated by the scalar Douglas-Kroll-Hess (DKH) Hamiltonian ${ }^{17}$ and the relativistic effective core potential (RECP), ${ }^{18}$ a conclusion that is further supported by the full four-component relativistic coupled cluster (CC) calculations of the dipole polarizability of the $\mathrm{Yb}$ atom. ${ }^{19}$ Since the asymptotic SO splitting in $\mathrm{M}_{\mathrm{II}}$ itself is small $\left(0.34 \mathrm{~cm}^{-1}\right),{ }^{20}$ the corresponding SO states can be obtained perturbatively by diagonalizing $\hat{H}_{\mathrm{el}}+\hat{H}_{\mathrm{SO}}$ in the $\Lambda-\Sigma$ basis, where $\hat{H}_{\mathrm{el}}$ is the nonrelativistic Born-Oppenheimer molecular electronic Hamiltonian, $\hat{H}_{\text {SO }}$ is the full microscopic Breit-Pauli SO operator, ${ }^{21}$ and $\Lambda$ and $\Sigma$ are the projections of the electronic orbital angular momentum and spin on the molecular axis. In the present case, a $26 \times 26$ SO Hermitian matrix is constructed. The diagonal matrix elements are composed of seven spin-free (SF) electronic states, $1{ }^{2} \Sigma^{+}, 2{ }^{2} \Sigma^{+}, 1{ }^{2} \Pi, 3^{2} \Sigma^{+}, 2{ }^{2} \Pi, 1^{4} \Sigma^{+}$, and $1^{4} \Pi$ originating from $\mathrm{M}_{\mathrm{I}}, \mathrm{M}_{\mathrm{II}}$, and $\mathrm{M}_{\mathrm{III}}$. The off-diagonal terms are given by the corresponding complex spin-orbit matrix elements (the diagonal elements of $\hat{H}_{\mathrm{SO}}$ are zero in the chosen basis). Diagonalization of this matrix yields the adiabatic SO states, which occur in 13 pairs of degenerate Kramer doublets. In the present study, SOCs were evaluated using the all-electron third-order DKH3 approach. As in the RECP approach, the corresponding one-electron effective SO potential has not been developed; therefore, no SO calculation was performed with the RECP.

\section{B. Electron correlation methods}

The spin unrestricted coupled-cluster method with single, double, and perturbative triple excitations $[\mathrm{UCCSD}(\mathrm{T})]^{22,23}$ was employed to calculate the ground state PEC and the electric field response properties. The reference wave function in $\mathrm{CC}$ calculations is from the spin restricted Hartree-Fock (ROHF) method. Twenty seven electrons, including $1 s^{2} 2 s^{1}$ of the $\mathrm{Li}$ atom and $5 s^{2} 5 p^{6} 4 f^{14} 6 s^{2}$ of the $\mathrm{Yb}$ atom, were treated explicitly in the $\operatorname{UCCSD}(\mathrm{T})$ calculations. Our previous study of the dipole polarizability suggests that the inner core electrons of the $\mathrm{Yb}$ atom $\left(4 s^{2} 4 p^{6} 4 d^{10}\right)$ marginally contribute. The PECs of the two quartet states were computed using the same UCCSD(T) method.

The calculations of the electronic excited states have been performed with the internally contracted multireference configuration interaction with single and double excitations ${ }^{24}$ plus the multireference version of the Davidson correction, ${ }^{25}$ denoted as MRCISD $(\mathrm{Q})$. The reference wave functions of the $\operatorname{MRCISD}(\mathrm{Q})$ calculation are obtained from a complete active space self-consistent field (CASSCF) approach. ${ }^{26}$ The active space in the CASSCF includes three electrons in eight molecular orbitals (MOs). The three electrons are the valence electrons of the LiYb molecule and the eight MOs are formed by the linear combination of $6 s 6 p$ orbitals of $\mathrm{Yb}$ and $2 s 2 p$ orbitals of Li. Twenty seven electrons were correlated. The $5 s 5 p 4 f$ orbitals of $\mathrm{Yb}$ and the $1 s$ orbital of $\mathrm{Li}$ do not form part of the active space, but they were correlated through single and double excitations. For the calculation of the $2^{2} \Sigma^{+}$and $1{ }^{2} \Pi$ states, a state-specific (SS) CASSCF calculation was carried out. As for the $3^{2} \Sigma^{+}$and $2{ }^{2} \Pi$ states, a state-averaged (SA) CASSCF including all five spin-free doublet states with equal weights was adopted. The effects due to the SS-CASSCF and SA-CASSCF reference wave functions will be addressed in Sec. III. The SOC matrix elements were evaluated at the same MRCISD level of theory based on a SA-CASSCF reference wave function. All seven SF states were included in the SA-CASSCF calculation with equal weights. MOLPRO 2009.1 suite of the quantum chemistry programs were used for all the electronic structure calculations. $^{27}$

\section{Basis sets}

Two sets of basis functions corresponding to the two approximate relativistic approaches, DKH and RECP, respectively, were applied. In the RECP approach, we adopted the same pseudopotentials, ECP28MDF, ${ }^{18}$ that was used earlier. ${ }^{16}$ It is a fully relativistic energy-adjusted pseudopotential that replaces the $1 s-3 d$ core orbitals of the $\mathrm{Yb}$ atom. A fully uncontracted $(15 s 15 p 12 d 11 f 9 g 3 h 2 i)$ basis set $^{16}$ was taken for the valence electrons. For the $\mathrm{Li}$ atom, an augmented polarized core-valence quintuple-zeta (aug-ccpCV5Z) basis set ${ }^{28}$ was employed. This rather extensive basis set has been used to evaluate the ground state PEC and electric and optical fields response properties at the $\mathrm{UCCSD}(\mathrm{T})$ level of theory. It serves as a benchmark for the all-electron DKH calculations, which determine the excited state PECs.

In the all-electron third-order DKH3 calculation, we used a relativistic atomic natural orbital basis (RCC-ANO) $(25 s 22 p 15 d 11 f 4 g 2 h) /[11 s 10 p 8 d 7 f 4 g 2 h]$, developed by Roos et $a l .{ }^{29}$ for the $\mathrm{Yb}$ atom. For the Li atom, DKH recontraction of the augmented polarized valence quadruple-zeta (aug-cc-pVQZ-DK) basis set ${ }^{30}$ was used. A comparison of these two approaches will be discussed in Sec. III C.

Due to the slow convergence to the basis set limit, basis sets superposition error (BSSE) may be significant in the determination of the PECs and other properties. ${ }^{31}$ Especially in the case of RECP calculations, a large set of uncontracted functions was used. To examine the effects due to the BSSE, the counterpoise $(\mathrm{CP})$ corrections $^{32}$ were employed for both the ground and excited states. The interaction energy $\Delta E$ at internuclear separation $R$ follows from

$\Delta E(R)=E_{A B}(R)-E_{A(B)}(R)-E_{B(A)}(R)-\left(E_{A B}(\infty)-E_{A}-E_{B}\right)$,

where $E_{A B}$ is the dimer energy, $E_{A(B)}$ is the monomer energy of $A$ calculated with the complete basis set of the dimer, and $E_{A}$ and $E_{B}$ are the energies in the monomer basis set only. For MRCI calculations, the physical picture is more complicated due to the size-extensivity problem of the CI method (although partially eliminated by the Davidson correction) 
and the configuration set superposition error (CSSE) caused by the incomplete description of the so-called Pauliforbidden states in the monomer calculation. ${ }^{31,33} \mathrm{~A}$ higherlevel CI description for monomer $A$ or $B$ than for the dimer $A B$ was recommended to alleviate the CSSE. ${ }^{33}$ In the present MRCISD calculations of the monomer, we allowed the $5 s 5 p 4 f$ orbitals of $\mathrm{Yb}$ and the $1 s$ orbital of $\mathrm{Li}$ to form the active space.

\section{Electric dipole moment}

The electric dipole moment $\mu$ is defined as the sum over all the charged particles, including the nuclei, of the products of the position vectors $\overrightarrow{\mathbf{r}}_{i}$ and the charges $q_{i}$. If $\Psi$ is the normalized wave function,

$$
\mu=\left\langle\Psi\left|\sum_{i=1}^{N} q_{i} \overrightarrow{\mathbf{r}}_{i}\right| \Psi\right\rangle
$$

The dipole moment also can be derived from the response of the molecular system to a weak external electric field $\mathcal{E}$ by

$$
E(\mathcal{E})=E(\mathcal{E}=0)-\mu_{i} \mathcal{E}_{i}-\frac{1}{2} \alpha_{i j} \mathcal{E}_{i} \mathcal{E}_{j}-\ldots
$$

The above equation contains implied sums over repeated indices. The first derivative of energy $E$ with respect to the external electric field $\mathcal{E}$ at $\mathcal{E}=0$ is the static dipole moment $\mu$ and the second derivative gives the static dipole polarizability tensor $\alpha_{i j}$. Equivalent results are expected when the approximate wave function $\Phi \cong \Psi$. However, in many cases, results can differ appreciably. Since the error in $E$ enters as the second order of the perturbation parameter $^{34}$ and the error in $\Phi$ is first order, the finite field (FF) procedure in Eq. (3) is preferred.

In the present study, $\mu$ was calculated by both methods at the MRCISD and UCCSD(T) levels. In the FF calculation, the dipole field strengths after a few tests were chosen as \pm 0.0003 , \pm 0.0006 , and \pm 0.0012 a.u. The dipole moment and the dipole polarizability were then derived from a polynomial fit to the computed field-dependent energies followed by the numerical derivatives.

\section{E. Long-range dispersion coefficients}

The accurate description of the long-range interaction is critical to the study of the dynamics in cold and ultracold conditions. The correct dispersion coefficient will also help to calibrate the electronic structure calculations for large internuclear separations. In our previous study, we derived for the $\mathrm{Yb}$ atom the frequency-dependent polarizability using the linear response CCSD method, ${ }^{16}$ and an accurate spectrum of $\mathrm{Li}$ atom has been recently reported in a variational calculation using Hylleraas coordinates. ${ }^{35}$ The leading dispersion coefficient $C_{6}$ for the ground $1^{2} \Sigma^{+}$state can then be obtained from the Casimir-Polder equation

$$
C_{6}=\frac{3}{\pi} \int_{0}^{\infty} d \omega \alpha_{A}(i \omega) \alpha_{B}(i \omega),
$$

where $\alpha(i \omega)$ is the dynamic polarizability at imaginary frequency $i \omega$.
For the $2{ }^{2} \Sigma^{+}$and $1{ }^{2} \Pi$ states, Eq. (4) cannot be applied directly because of the downward transition, ${ }^{2} \mathrm{P}$ to ${ }^{2} \mathrm{~S}$, in the $\mathrm{Li}$ atom. A correction can be made to separate it out ${ }^{36}$ in the integrand of Eq. (4) or we may calculate $\mathrm{C}_{6}$ by the sum-overoscillator strengths. For the $n s-n p$ configuration, ${ }^{37}$

$$
\begin{aligned}
C_{6}= & \sum_{n_{A}, n_{B}} F^{P-S} \frac{f_{A}^{P-S} f_{B}^{S-P}}{\Delta E_{A}^{P-S} \Delta E_{B}^{S-P}\left(\Delta E_{A}^{P-S}+\Delta E_{B}^{S-P}\right)} \\
& +\sum_{n_{A}, n_{B}} F^{P-P} \frac{f_{A}^{P-P} f_{B}^{S-P}}{\Delta E_{A}^{P-P} \Delta E_{B}^{S-P}\left(\Delta E_{A}^{P-P}+\Delta E_{B}^{S-P}\right)} \\
& +\sum_{n_{A}, n_{B}} F^{P-D} \frac{f_{A}^{P-D} f_{B}^{S-P}}{\Delta E_{A}^{P-D} \Delta E_{B}^{S-P}\left(\Delta E_{A}^{P-D}+\Delta E_{B}^{S-P}\right)},
\end{aligned}
$$

where $n_{A}$ and $n_{B}$ are the number of dipole allowed transitions and $f_{A}^{L_{1}-L_{2}}$ and $\Delta E_{A}^{L_{1}-L_{2}}$ are, respectively, the oscillator strengths and transition energies for the $L_{1} \rightarrow L_{2}$ transition, where $L_{1}$ and $L_{2}$ are the electronic angular momenta of the corresponding atomic states. The associated angular coefficients $F^{P-S}, F^{P-P}$, and $F^{P-D}$, for the $\Pi$ state are $3 / 4,15 / 8$, and $57 / 40$, and for the $\Sigma^{+}$state are $3,3 / 4$, and 33/20, respectively.

The atomic dipole oscillator strengths of the $\mathrm{Yb}$ atom were determined by the linear response density function theory (DFT) as implemented in the Dalton quantum chemistry program. ${ }^{38}$ The hybrid form of Perdew-BurkeErnzerhof functional (PBE0) (Ref. 39) was adopted for the DFT calculation and the RECP approach was used.

\section{F. Spectroscopic constants and Franck-Condon factors}

PECs of the ground and excited states were fitted to the $a b$ initio points from $R=3.0$ to $R=20.0 a_{0}$ by the analytical form

$$
V(R)=\sum_{i=0}^{8} a_{i} R^{i} e^{-\alpha(R-\beta)}-\frac{1}{2}[1+\tanh (\gamma+\lambda R)] \sum_{n=0}^{4} \frac{C_{2 n+6}}{R^{2 n+6}} .
$$

The coefficients $C_{6}$ were fixed at our derived values. The nonlinear parameters $\alpha, \beta, \gamma$, and $\lambda$, and the linear $a_{i}$ and $C_{n}(n \neq 6)$ parameters were fully optimized using the Levenberg-Marquardt nonlinear least square algorithm. The radial Schrödinger equation for the nuclear motion was then solved using the Fourier grid Hamiltonian method. ${ }^{40}$ A nonlinear least square fitting of the computed vibrational eigenvalues to the truncated anharmonic oscillator

$$
E(\nu)=\omega_{e}\left(\nu+\frac{1}{2}\right)-\omega_{e} x_{e}\left(\nu+\frac{1}{2}\right)^{2}+\omega_{e} y_{e}\left(\nu+\frac{1}{2}\right)^{3}
$$

yielded vibrational spectroscopic constants $\omega_{e}, \omega_{e} x_{e}$, and $\omega_{e} y_{e}$. The Franck-Condon (FC) overlaps were evaluated from the corresponding vibrational eigenfunctions.

\section{RESULTS AND DISCUSSION}

\section{A. Long-range dispersion coefficients}

The calculated leading dispersion coefficients $C_{6}$ for the ground and excited states of the LiYb molecule are listed in Table I. The DFT and the CCSD results are also given for 
TABLE I. Long-range dispersion coefficients (a.u.).

\begin{tabular}{lcc}
\hline \hline State & DFT (PBE0) & CCSD \\
\hline LiYb $1{ }^{2} \Sigma^{+}$ & 1606 & 1594 \\
LiYb $2{ }^{2} \Sigma^{+}$ & 5994 & $\ldots$ \\
LiYb $1^{2} \Pi$ & 2062 & $\ldots$ \\
$\mathrm{Yb}_{2}{ }^{1} \Sigma^{+}$ & 2058 & 2062 \\
\hline \hline
\end{tabular}

$\mathrm{Yb}_{2}$ and presented in Table I. The agreement between the two methods is to within $\sim 1 \%$. This is encouraging because the PBE0 functionals could be used to study the interactions of two or more $\mathrm{LiYb}$ molecules. The derived $C_{6}$ of the ground state is 1606 a.u., whereas an empirical estimate of the $C_{6}$ using atomic polarizabilities ${ }^{41}$ yields 1610 a.u. The two excited states associated with $\mathrm{M}_{\mathrm{II}}$ have quite different interaction strengths; the $C_{6}$ for the $2{ }^{2} \Sigma^{+}$state is 5994 a.u. and for the $1{ }^{2} \Pi$ state is just 2062 a.u. We did not study the long-range interactions associated with the $\mathrm{Yb}\left({ }^{3} \mathrm{P}\right)$ atom, where more rigorous relativistic treatments are necessary.

\section{B. Atomic data}

The accuracy of the calculations is tested by comparisons with experimental atomic spectra. The computed spinorbital levels of the $\mathrm{Li}$ and $\mathrm{Yb}$ atoms are compiled in Table II. For the $\mathrm{Li}$ atom, the SA-MRCI calculation using a SA $\left[\operatorname{Li}\left({ }^{2} \mathrm{~S}\right)\right.$ and $\operatorname{Li}\left({ }^{2} \mathrm{P}\right)$ with equal weights and three electrons in five orbitals] CASSCF reference wave functions shows an energy separation of $14821.40 \mathrm{~cm}^{-1}$ between $\operatorname{Li}\left({ }^{2} \mathrm{~S}\right)$ and $\mathrm{Li}\left({ }^{2} \mathrm{P}_{1 / 2}\right)$ and a SO splitting of $0.25 \mathrm{~cm}^{-1}$ for $\operatorname{Li}\left({ }^{2} \mathrm{P}\right)$. The error in the energy separation, compared with the observed value of $14903.66 \mathrm{~cm}^{-1}$, is less than $1 \%$, but the error associated with the SO splitting is relatively large, $0.09 \mathrm{~cm}^{-1}$ smaller than the experiment data of $0.34 \mathrm{~cm}^{-1}$. These results can be improved by using more optimal and larger basis sets, but at computational expense.

For the $\mathrm{Yb}$ atom, the reference CASSCF wave function was constructed by averaging $\mathrm{Yb}\left({ }^{1} \mathrm{~S}\right)$ and $\mathrm{Yb}\left({ }^{3} \mathrm{P}\right)$ states with equal weights and by distributing two $6 s$ electrons among four atomic orbitals $(6 s 6 p)$. The following SA-MRCI calculations correlate the same number of electrons (24) as in the molecular calculation. The derived $\mathrm{SO}$ levels between $\mathrm{Yb}\left({ }^{1} \mathrm{~S}\right)$ and $\mathrm{Yb}\left({ }^{3} \mathrm{P}\right)$ states in general are $\sim 8 \%$ smaller than experimental results. The computed SO splitting of $969.7 \mathrm{~cm}^{-1}$ between $\mathrm{Yb}\left({ }^{3} \mathrm{P}_{0}\right)$ and $\mathrm{Yb}\left({ }^{3} \mathrm{P}_{1}\right)$ is overestimated
TABLE II. The comparisons between experimental and theoretical derived spin-orbit levels $\left(\right.$ in $\mathrm{cm}^{-1}$ ) for the $\mathrm{Li}$ and $\mathrm{Yb}$ atoms.

\begin{tabular}{lccc}
\hline \hline & Theory $^{\mathrm{a}}$ & Theory $^{\mathrm{b}}$ & Expt. \\
\hline $\mathrm{Li}\left({ }^{2} \mathrm{~S}\right)$ & 0.0 & & 0.0 \\
$\mathrm{Li}\left({ }^{2} \mathrm{P}_{1 / 2}\right)$ & 14821.40 & & 14903.66 \\
$\mathrm{Li}\left({ }^{2} \mathrm{P}_{3 / 2}\right)$ & 14821.65 & & 14904.00 \\
$\mathrm{Yb}\left({ }^{1} \mathrm{~S}\right)$ & 0.0 & 0.0 & 0.0 \\
$\mathrm{Yb}\left({ }^{3} \mathrm{P}_{0}\right)$ & 15968.0 & 16821.9 & 17288.439 \\
$\mathrm{Yb}\left({ }^{3} \mathrm{P}_{1}\right)$ & 16937.7 & 17788.6 & 17992.007 \\
$\mathrm{Yb}\left({ }^{3} \mathrm{P}_{2}\right)$ & 18868.2 & 19722.1 & 19710.388 \\
\hline \hline
\end{tabular}

${ }^{\mathrm{a}}$ The spin-orbit couplings are evaluated using the SA-MRCISD wave function and the spin-free energy is calculated at the SA-MRCISD(Q) level of theory.

${ }^{\mathrm{b}} \mathrm{Same}$ as a except that the spin-free energy is replaced by the $\operatorname{UCCSD}(\mathrm{T})$ result.

by $266 \mathrm{~cm}^{-1}$ and the splitting between $\mathrm{Yb}\left({ }^{3} \mathrm{P}_{0}\right)$ and $\mathrm{Yb}\left({ }^{3} \mathrm{P}_{2}\right)$ is larger by $478 \mathrm{~cm}^{-1}$. If we replace the SF SA-MRCI $\mathrm{Yb}\left({ }^{1} \mathrm{~S}\right)$ and $\mathrm{Yb}\left({ }^{3} \mathrm{P}\right)$ energy separation by the $\mathrm{UCCSD}(\mathrm{T})$ derived value of $18842 \mathrm{~cm}^{-1}$, the errors in energy separation between $\mathrm{Yb}\left({ }^{1} \mathrm{~S}\right)$ and the levels in $\mathrm{Yb}\left({ }^{3} \mathrm{P}\right)$ are greatly reduced to less than $3 \%$.

In a recent calculation of the $\mathrm{RbYb}$ molecule, close agreement with the experiment for the SO levels of $\mathrm{Yb}\left({ }^{3} \mathrm{P}\right)$ and $\mathrm{Yb}\left({ }^{1} \mathrm{~S}\right)$ was reported ${ }^{42}$ using a similar MRCI treatment but with a four-component Dirac-Coulomb Hamiltonian. For the $\mathrm{Yb}$ atom, only a qualitative picture can be obtained with the state interacting strategy for the SOC. Our primary interest are the states associated with the $\operatorname{Li}\left({ }^{2} \mathrm{P}\right)$ asymptote and the states correlating to the $\mathrm{Yb}\left({ }^{3} \mathrm{P}\right)$ asymptote contribute perturbatively.

\section{Ground state spin-free PEC and BSSE correction}

We benchmark the computational approaches with and without CP corrections to the PEC of the ground state of LiYb. The potential depth $D_{e}$ and equilibrium distance $R_{e}$ obtained from CCSD(T), SS-MRCI, and SA-MRCI methods with and without the CP corrections are summarized in Table III. In addition, an effective long-range dispersion coefficient $\widetilde{C}_{6}=\Delta E \times R^{6}$ at $R=20.0 a_{0}$ is also given in Table III, where $\Delta E$ is the interaction energy relative to $\mathrm{M}_{\mathrm{I}}$.

For the LiYb molecule, the ground state is largely dominated by a single configuration, as indicated by the leading CI coefficient $(>0.95)$ in the MRCI wave function and the

TABLE III. The potential depth $D_{e}\left(\mathrm{~cm}^{-1}\right)$, equilibrium distance $R_{e}\left(a_{0}\right)$, and effective dispersion coefficient $\widetilde{C}_{6}$ (a.u.) of the ground state $\mathrm{LiYb}$ molecule calculated at the coupled-cluster and the multireference configuration interaction levels of theory with the relativistic pseudopotential and all-electron Douglas-Kroll approximations. The $\widetilde{C}_{6}$ is obtained at $R=20.0 a_{0}$.

\begin{tabular}{lcccc}
\hline \hline & CCSD $(\mathrm{T}) / \mathrm{ECP}$ & $\mathrm{CCSD}(\mathrm{T}) / \mathrm{ANO}$ & SS-MRCI/ANO & SA-MRCI/ANO \\
\hline$D_{e}$ (with CP) & 1642 & 1545 & 1438 & 1112 \\
$D_{e}$ (without CP) & 1701 & 1733 & 1607 & 1278 \\
$R_{e}$ (with CP) & 6.65 & 6.71 & 6.70 & 6.80 \\
$R_{e}$ (without CP) & 6.65 & 6.60 & 6.61 & 6.70 \\
$\tilde{C}_{6}$ (with CP) & 1766 & 1804 & 1424 & 1677 \\
$\widetilde{C}_{6}$ (without CP) & 2025 & 2284 & 1846 & 2250 \\
\hline \hline
\end{tabular}


TABLE IV. The potential depth $D_{e}\left(\mathrm{~cm}^{-1}\right)$, equilibrium distance $R_{e}\left(a_{0}\right)$, vertical excitation energy $T_{v}\left(\mathrm{~cm}^{-1}\right)$, adiabatic excitation energy $T_{e}\left(\mathrm{~cm}^{-1}\right)$, and effective dispersion coefficient $\widetilde{C}_{6}$ (a.u.) of the excited states of the $\mathrm{LiYb}$ molecule calculated at the coupled-cluster and the multireference configuration interaction levels of theory with the all-electron Douglas-Kroll approximations. The $\widetilde{C}_{6}$ is obtained at $R=20.0 a_{0}$. The tabulated values are without (with) $\mathrm{CP}$ corrections.

\begin{tabular}{lcccccc}
\hline \hline State & Method & $R_{e}$ & $D_{e}$ & $T_{e}$ & $T_{v}$ & $\tilde{C}_{6}$ \\
\hline $2^{2} \Sigma^{+}$ & SA-MRCISD(Q) & $6.50(6.50)$ & $4951(4757)$ & 11541 & 11498 & $5077(4531)$ \\
$2^{2} \Sigma^{+}$ & SS-MRCISD(Q) & 6.50 & 5651 & $10798^{\mathrm{a}}$ & $10845^{\mathrm{a}}$ & 5075 \\
$1^{2} \Pi$ & SA-MRCISD(Q) & $5.60(5.70)$ & $9198(8897)$ & 6687 & 8594 & $1894(1351)$ \\
$1^{2} \Pi$ & SS-MRCISD(Q) & 5.60 & 9494 & $6955^{\mathrm{a}}$ & $8660^{\mathrm{a}}$ & 1590 \\
$3^{2} \Sigma^{+}$ & SA-MRCISD(Q) & $7.30(7.40)$ & $4898(4767)$ & $15070^{\mathrm{b}}$ & $15254^{\mathrm{b}}$ & $\ldots$ \\
$2^{2} \Pi$ & SA-MRCISD(Q) & $6.00(6.00)$ & $4919(4682)$ & $15047^{\mathrm{b}}$ & $15663^{\mathrm{b}}$ & $\ldots$ \\
$1^{4} \Sigma^{+}$ & ROHF-UCCSD(T) & $8.62(8.60)$ & $781(646)$ & 19321 & 20209 & $\ldots$ \\
$1^{4} \Pi$ & ROHF-UCCSD(T) & $6.20(6.30)$ & $4466(4129)$ & 16271 & 15985 & $\cdots$ \\
\hline \hline
\end{tabular}

${ }^{2}$ Energies are derived based on the shifted asymptotic energy separation of $14904 \mathrm{~cm}^{-1}$.

${ }^{b}$ Energies are derived based on the shifted asymptotic energy separation of $18422 \mathrm{~cm}^{-1}$.

small $(<0.03) T_{1}$ diagnostic $^{43}$ in the $\mathrm{CC}$ calculations for the internuclear distances $R$ beyond $4.0 a_{0}$. The $\operatorname{CCSD}(\mathrm{T})$ results from the RECP and DKH3 approaches agree closely with each other. A similar value for $R_{e}$ between 6.7 and $6.8 a_{0}$ is predicted in both methods and the $\mathrm{CP}$ correction is small. Values to within $1 \%$ for the potential depth $D_{e}$ are obtained without the CP corrections. With the $\mathrm{CP}$ corrections, $D_{e}$, using the RECP approach, is about $97 \mathrm{~cm}^{-1}$ deeper than that obtained with the DKH3 approximation. Inspecting the effective $\widetilde{C}_{6}$, both methods with the CP corrections predict reasonable values (1766 and 1804 a.u.) that are close to the linear response prediction of 1606 a.u. $\widetilde{C}_{6}$ without $\mathrm{CP}$ corrections is larger by more than $15 \%$. It is difficult to establish which result is superior. The polarization functions in the RECP approach were added by scaling the corresponding lower angular momentum functions ${ }^{16}$ and are therefore not optimal. The all-electron RCC-ANO basis, on the contrary, was obtained through the optimization by a multireference perturbation calculation. The scalar relativistic effects are adequately described in both methods. Thus, it is likely that the results from the DKH3 approach with the ANO-RCC basis are more reliable and it was adopted in the calculations of the excited state PEC and SOC.

The potential depth from the MRCI calculation is, in general, underestimated. The $D_{e}=1112 \mathrm{~cm}^{-1}$ from the SA-MRCI calculation is undervalued by $\sim 400 \mathrm{~cm}^{-1}$. This is mainly due to the poor description of the ground state wave function by the state-averaged optimization of the molecular orbital, as indicated by the better agreement between the SSMRCI and CCSD(T) methods. The SS-MRCI method improves the results but still underestimates the potential depth by $\sim 150 \mathrm{~cm}^{-1}$ for both of the results with and without $\mathrm{CP}$ corrections. Note that the $\mathrm{CP}$ uncorrected value from MRCI calculations agrees better with the $\operatorname{CCSD}(\mathrm{T}) \mathrm{CP}$ corrected results. Especially, the SS-MRCI value $1607 \mathrm{~cm}^{-1}$ is $\sim 3 \%$ higher than the CP corrected CCSD(T) value of $1545 \mathrm{~cm}^{-1}$. Therefore, the SS-MRCI results without CP corrections are more reliable.

\section{Spin-free excited states PECs}

PECs of the doublet excited states are determined by employing the SA-MRCI and the SS-MRCI methods. The
$\mathrm{UCCSD}(\mathrm{T})$ theory has been employed to obtain the PECs of the quartet states. All calculations adopt the ANO-RCC basis with the DKH3 Hamiltonian. The derived spectroscopic results are listed in Table IV and the PECs are shown in Fig. 1.

Similar to the ground state, the potential depths of the $2^{2} \Sigma^{+}$and $1{ }^{2} \Pi$ states from the SA-MRCI calculations are smaller than those from the SS-MRCI method. The effective $\tilde{C}_{6}$ are calculated for these two states at $R=20.0 a_{0}$ and are given in Table IV. Both values of $\widetilde{C}_{6}$ (with and without $\mathrm{CP}$ corrections) are smaller than theoretical dispersion coefficients derived from the linear response theory and listed in Table I, but those without the $\mathrm{CP}$ corrections are closer to the predictions by the linear response theory. The equilibrium distance is less sensitive to BSSE. These results indicate that MRCI methods underestimate the interaction energy, which BSSE may partially compensate. Therefore, the SS-MRCI results without the $\mathrm{CP}$ corrections are more reliable. The potential depths $D_{e}$ are 5651 and $9494 \mathrm{~cm}^{-1}$ for the $2{ }^{2} \Sigma^{+}$and $1{ }^{2} \Pi$ states, respectively. Deeper potential and smaller longrange interaction in the $1^{2} \Pi$ state result in a crossing with

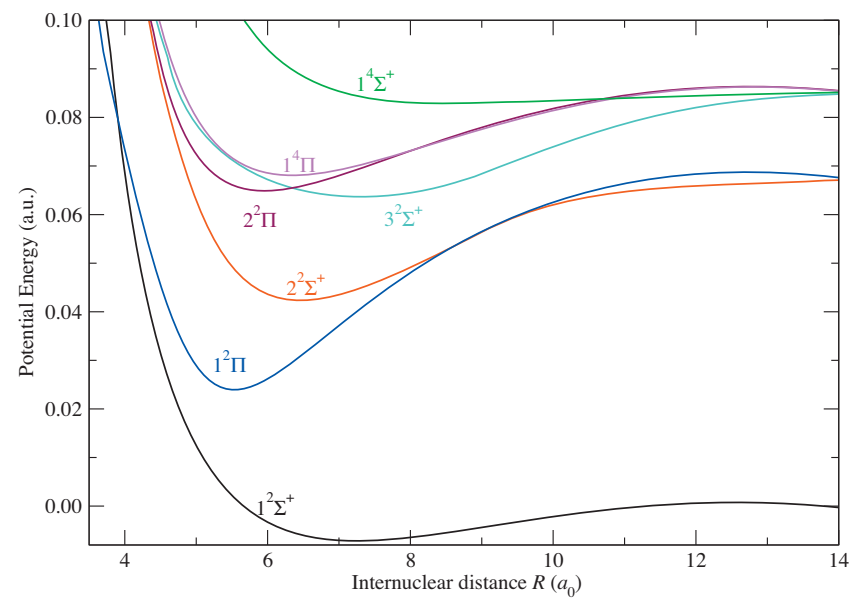

FIG. 1. Spin-free potential energy curves of the ground and excited states of the LiYb molecule. The $1^{2} \Sigma^{+}$state is determined by the CP corrected DKH3-UCCSD(T) calculation. The $2{ }^{2} \Sigma^{+}, 1{ }^{2} \Pi, 3^{2} \Sigma^{+}$, and $2{ }^{2} \Pi$ states are computed at the MRCISD(Q) level of theory without the CP correction and the quartet states are derived from the CP corrected DKH3-UCCSD(T) calculations. 
the $2{ }^{2} \Sigma^{+}$state around $8.5 a_{0}$. Asymptotically, these two states are degenerate and the calculated energy separation (at the SA-MRCI level) from $\mathrm{M}_{\mathrm{I}}, 14807 \mathrm{~cm}^{-1}$, is smaller than the experimental value of $14904 \mathrm{~cm}^{-1}$. The vertical $\left(T_{v}\right)$ and adiabatic $\left(T_{e}\right)$ excitation energies to the $1^{2} \Pi$ state given by the SA-MRCI calculations are 8594 and $6887 \mathrm{~cm}^{-1}$.

If we replace the two SA-MRCI potentials with the results of SS-MRCI calculations and shift them to match the correct asymptotic energy separation, $T_{v}$ and $T_{e}$ become 8660 and $6955 \mathrm{~cm}^{-1}$, respectively. They are not very different from the SA-MRCI predictions. The corresponding best values for the $2^{2} \Sigma$ state are 10845 and $10798 \mathrm{~cm}^{-1}$. For such energy separations, the spectrum of the ground state will not be perturbed strongly by the SO interaction.

The SS-MRCI calculations for the $3{ }^{2} \Sigma^{+}$and $2{ }^{2} \Pi$ states were not successful. Trial calculations with increased weights on these two states at selected $R$ showed a similar tendency for the change of the potential depth. But the CASSCF convergence became problematic and we did not perform the SS-MRCI calculations for the PECs correlating to $\mathrm{M}_{\mathrm{III}}$. Nor were the $\mathrm{CP}$ corrections applied, as suggested by the results from the ground and lower excited states. Neither state is deeply bound; $D_{e}=4897 \mathrm{~cm}^{-1}$ for the $3{ }^{2} \Sigma^{+}$state and $D_{e}=4919 \mathrm{~cm}^{-1}$ for the $2^{2} \Pi$ state.

The quartet states $1^{4} \Sigma^{+}$and $1^{4} \Pi$ are determined more precisely at the $\operatorname{UCCSD}(\mathrm{T})$ level of theory with $\mathrm{CP}$ corrections applied. The $1{ }^{4} \Sigma^{+}$state is weakly bound, $D_{e}=781 \mathrm{~cm}^{-1}$. The $1{ }^{4} \Pi$ state has a deeper potential well, $D_{e}=4466 \mathrm{~cm}^{-1}$. There are extensive crossings among these SF doublet and quartet states. As a result, avoided crossings are expected among the corresponding SO states. Asymptotically, the $\operatorname{UCCSD}(\mathrm{T})$ energy separation to $M_{\mathrm{I}}$ is $18842 \mathrm{~cm}^{-1}$. We again shift the PECs of the $3^{2} \Sigma^{+}$and $2^{2} \Pi$ states to agree with this value. The energy separation between $\mathrm{M}_{\mathrm{II}}$ and $\mathrm{M}_{\mathrm{III}}$ is $3538 \mathrm{~cm}^{-1}$, and it increases as $R$ decreases. In the equilibrium region, the smallest adiabatic energy separation between the $2^{2} \Pi$ and $2^{2} \Sigma^{+}$states is about $4274 \mathrm{~cm}^{-1}$.

\section{E. Nonadiabatic interactions between the two excited ${ }^{2} \Sigma^{+}$states}

There is an avoided crossing between the $2^{2} \Sigma^{+}$and $3^{2} \Sigma^{+}$states at $\sim 9.0 a_{0}$. It is manifested by the increased mixing of the CI coefficients in the SA-MRCI wave functions in this region. The energy gap in this mixing region is $\sim 1500 \mathrm{~cm}^{-1}$ and hence the rovibrational perturbation is expected to be small. A coupled-channel bound state calculation was carried out to investigate this effect.

The derivative coupling $\left\langle 2^{2} \Sigma^{+}|\partial / \partial R| 3^{2} \Sigma^{+}\right\rangle$was evaluated at the SA-MRCI level of theory using the DDR procedure implemented in MOLPRO. The result is plotted in Fig. 2 along with the spin-free PECs of the $2{ }^{2} \Sigma^{+}$and $3{ }^{2} \Sigma^{+}$states. The coupling peaks around $R=9.0 a_{0}$ and assumes a Lorentzian shape. The two-state coupled radial Schrödinger equations were numerically solved ${ }^{44}$ and the resolved rotationless vibration levels were compared to those obtained without the derivative coupling. For the $2^{2} \Sigma^{+}$state, the vibration levels near the threshold are shifted by less than $0.05 \mathrm{~cm}^{-1}$.

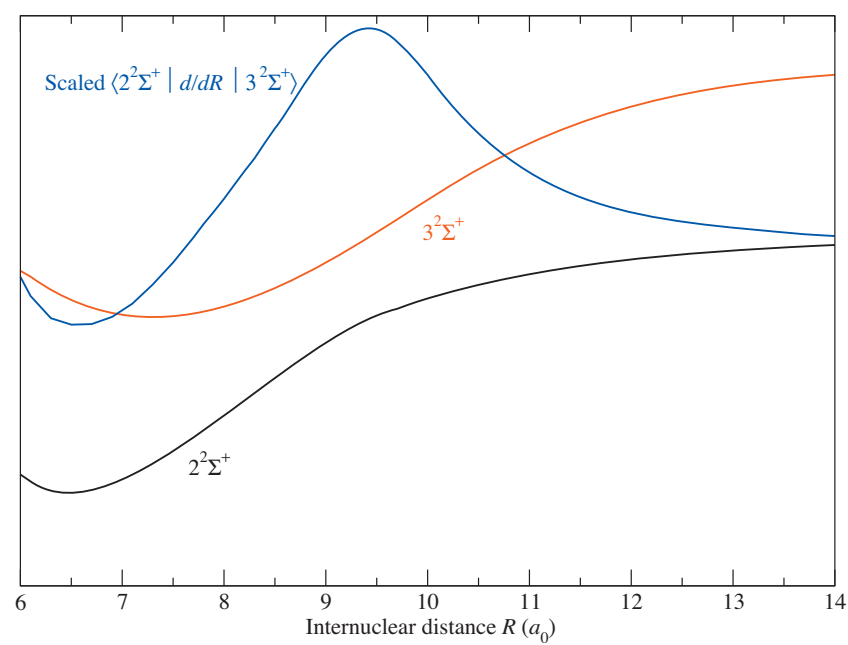

FIG. 2. Spin-free potential energy curves of the $2{ }^{2} \Sigma^{+}$and $3{ }^{2} \Sigma^{+}$states, and the scaled derivative coupling as a function of the internuclear distance $R$. All are computed at the SA-MRCISD(Q) level of theory.

\section{F. Spin-orbit coupled PECs for ground and lowest three excited states}

The SO states are obtained by the state interacting method and labeled by quantum numbers $\Omega=\Lambda+\Sigma$. The diagonal SF elements for each state are chosen as follows. For the ground state $\left(1^{2} \Sigma^{+}\right)$, the CP corrected UCCSD(T)/ ANO-RCC interaction potential is adopted. The SS-MRCI/ ANO-RCC results without the $\mathrm{CP}$ corrections are used for the $2^{2} \Sigma^{+}$and $1^{2} \Pi$ states. These two potentials are also shifted so that the asymptotic energy separation to the ground state equals the ${ }^{2} \mathrm{P}-{ }^{2} \mathrm{~S}$ separation of the $\mathrm{Li}$ atom. For the $1{ }^{4} \Sigma^{+}$and $1{ }^{4} \Pi$ states, we use the CP corrected UCCSD(T)/ANO-RCC data. Finally, for the $3{ }^{2} \Sigma^{+}$and $2{ }^{2} \Pi$ states, SA-MRCI values without the CP corrections are employed. Again, these two potentials are shifted to match the asymptotic energy separation of $18842 \mathrm{~cm}^{-1}$ between the ground and the quartet states. The off-diagonal SO matrix elements are computed with the full BP Hamiltonian using the SA-MRCI wave functions.

The SO PECs are displayed in Fig. 3 and the corresponding spectroscopic constants are summarized in Table V

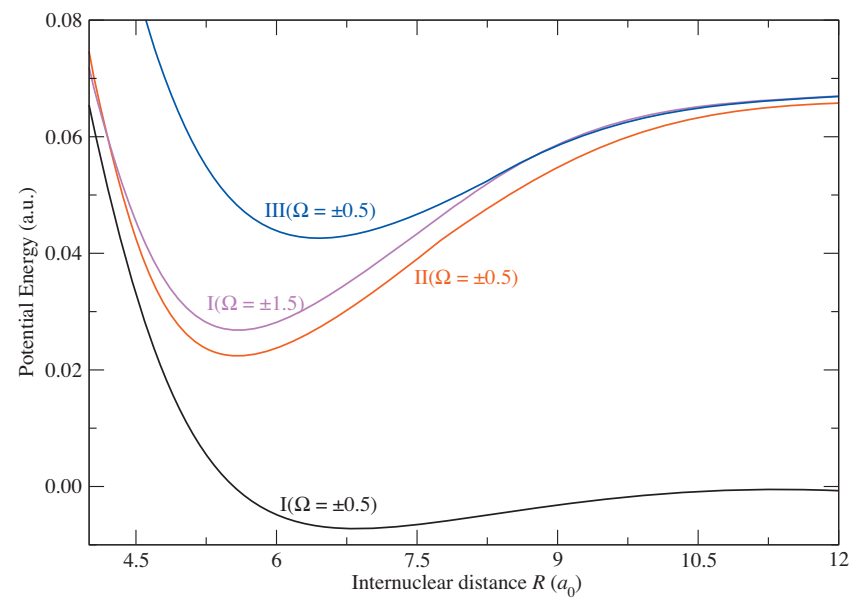

FIG. 3. Spin-orbit potential energy curves of the ground and three lowest excited states of the LiYb molecule. 
TABLE V. Spectroscopic constants for ground and three lowest excited states ( $\Omega$ designation) of the ${ }^{7} \mathrm{Li}{ }^{172} \mathrm{Yb}$ molecule.

\begin{tabular}{lcccccccc}
\hline \hline $\mathrm{N}(\Omega)$ & $\Lambda-\Sigma$ & $R_{e}\left(a_{0}\right)$ & $\begin{array}{c}\omega_{e}{ }^{\mathrm{a}} \\
\left(\mathrm{cm}^{-1}\right)\end{array}$ & $\begin{array}{c}\omega_{e} x_{e}{ }^{\mathrm{a}} \\
\left(\mathrm{cm}^{-1}\right)\end{array}$ & $\begin{array}{c}D_{e} \\
\left(\mathrm{~cm}^{-1}\right)\end{array}$ & $\begin{array}{c}B_{e}{ }^{\mathrm{b}} \\
\left(\mathrm{cm}^{-1}\right)\end{array}$ & $\begin{array}{c}T_{v} \\
\left(\mathrm{~cm}^{-1}\right)\end{array}$ & $\begin{array}{c}T_{e} \\
\left(\mathrm{~cm}^{-1}\right)\end{array}$ \\
\hline $\mathrm{I}\left(\frac{1}{2}\right)$ & $1^{2} \Sigma^{+}$ & 6.681 & 147.36 & 3.630 & 1577 & 0.198 & 0 & 0 \\
$\mathrm{II}\left(\frac{1}{2}\right)$ & $2^{2} \Sigma^{+}-1^{2} \Pi$ & 5.622 & 276.19 & 1.341 & 10008 & 0.282 & 8076 & 6473 \\
$\mathrm{I}\left(\frac{3}{2}\right)$ & $1^{2} \Pi$ & 5.619 & 274.20 & 1.354 & 9020 & 0.282 & 9049 & 7462 \\
$\mathrm{III}\left(\frac{1}{2}\right)$ & $1^{2} \Pi-2^{2} \Sigma^{+}$ & 6.473 & 210.82 & 0.960 & 5576 & 0.213 & 10956 & 10906 \\
\hline \hline
\end{tabular}

${ }^{a} \omega_{e}$ and $\omega_{e} x_{e}$ are obtained by fitting only the low-lying vibrational levels.

${ }^{\mathrm{b}} B_{e}$ is the inertial rotational constant for the ground vibrational state.

for the ground and lowest three excited states. These spectroscopic constants are derived for the isotopic combination of bosonic ${ }^{7} \mathrm{Li}$ and ${ }^{172} \mathrm{Yb}$ with zero rotational quantum number. The ground state $\mathrm{I}\left(\Omega=\frac{1}{2}\right)$ largely maintains its SF $1{ }^{2} \Sigma^{+}$ character. The long-range part of the potential changes little and the effective $\widetilde{C}_{6}$ at $R=20.0 a_{0}$ is the same as for the $\mathrm{SF}$ potential. The equilibrium distance $R_{e}=6.68 a_{0}$ remains virtually the same, but the potential depth $1577 \mathrm{~cm}^{-1}$ increases by $32 \mathrm{~cm}^{-1}$. A similar change due to the SOC was also found for the ground state $\mathrm{RbYb}$ molecule calculated at the full relativistic four-component $\operatorname{CCSD}(\mathrm{T})$ level of theory, ${ }^{42}$ but $\mathrm{RbYb}$ has a shallow well of $\sim 804 \mathrm{~cm}^{-1}$. The calculated $\mathrm{LiYb}$ potential supports 26 vibrational levels and the last bound state lies $0.013 \mathrm{~cm}^{-1}$ below $\mathrm{M}_{\mathrm{I}}$. Compared to the SF precursor, $\omega_{e}$ increases by $\sim 4 \mathrm{~cm}^{-1}$.

The first excited state $\operatorname{II}\left(\Omega= \pm \frac{1}{2}\right)$ originates from the mixing of the SF $1{ }^{2} \Pi$ and $2{ }^{2} \Sigma^{+}$states. At short range, it largely retains the original $1{ }^{2} \Pi$ character. Its depth $D_{e}$ $=10008 \mathrm{~cm}^{-1}$ is increased by $\sim 514 \mathrm{~cm}^{-1}$ from the SF potential. The nature of the potential changes to $2^{2} \Sigma^{+}$for $R$ $>8.5 a_{0}$. This is because of the crossing of the two SF states. With the inclusion of SOCs, both states yield the $|\Omega|=\frac{1}{2}$ components and the crossing disappears. Similar to the ground state, the long-range part of the $\operatorname{II}\left(\Omega= \pm \frac{1}{2}\right)$ potential is virtually the same as that in the SF counterpart, thanks to the small $\mathrm{Li}\left({ }^{2} \mathrm{P}\right)$ SO coupling and large energy separation from other states. Therefore, we adopt the $C_{6}$ from our nonrelativistic linear response calculations. The equilibrium distance of the potential has a slight increase of $\sim 0.02 a_{0}$. There are 66 vibrational levels and $\omega_{e}$ is almost the same as that of the SF $1{ }^{2} \Pi$ state. The last bound state positions at $0.078 \mathrm{~cm}^{-1}$ below the dissociation limit.

The second excited state is the II $\left(\Omega= \pm \frac{3}{2}\right)$ state. It retains mostly the original SF $1{ }^{2} \Pi$ character with small mixings from the upper $\Pi$ states. Its depth $D_{e}=9020 \mathrm{~cm}^{-1}$ is $474 \mathrm{~cm}^{-1}$ shallower than that of the original SF state. The long-range part of the potential remains largely the original $\mathrm{SF} 1{ }^{2} \Pi$ state, as it does at the equilibrium distance. The $\mathrm{II}\left(\Omega= \pm \frac{3}{2}\right)$ potential supports 56 vibrational levels and the last bound state lies $0.0069 \mathrm{~cm}^{-1}$ below the asymptote. Compared to the SF predecessor, $\omega_{e}=274.2 \mathrm{~cm}^{-1}$ is only decreased by about $2 \mathrm{~cm}^{-1}$.

The $\operatorname{III}\left(\Omega= \pm \frac{1}{2}\right)$ state is the third excited state. Contrary to the $\operatorname{II}\left(\Omega= \pm \frac{1}{2}\right)$ state, it has $2^{2} \Sigma^{+}$character at short range and $1{ }^{2} \Pi$ character for $R>8.5 a_{0}$. The potential depth $D_{e}$ $=5576 \mathrm{~cm}^{-1}$ is $75 \mathrm{~cm}^{-1}$ shallower than that of the $\mathrm{SF}^{2} \Sigma^{+}$ state. Again, the equilibrium distance is insensitive to SOC and it supports 44 vibrational structures with the last bound state positioned at $0.001 \mathrm{~cm}^{-1}$ below the dissociation limit. The harmonic term $\omega_{e}$ has a small decrease from 211.3 to $210.8 \mathrm{~cm}^{-1}$.

The higher excited SO states are not presented because the underlying SF results are less accurate and the SOCs with the next asymptote beyond $\mathrm{M}_{\mathrm{III}}$ are not considered.

\section{G. Franck-Condon overlaps}

The demonstration of simultaneous magnetic optical trap trapping of $\mathrm{Li}$ and $\mathrm{Yb}$ atoms holds promise for the eventual production of molecular species comprised of bosonic and fermionic mixtures. ${ }^{15}$ Photoassociation will likely be the preferred technique, for which favorable transitions are necessary. Our calculated FC factors, expressed as twodimensional intensity plots, are displayed in Fig. 4 as functions of the vibrational quantum numbers for the ground and excited states. For all three excited states involved in the $\mathrm{Li}\left({ }^{2} \mathrm{~S}\right)-\mathrm{Li}\left({ }^{2} \mathrm{P}\right)$ transition, large overlaps on the order of $10^{-1}-10^{-2}$ occur between the high-lying vibrational states. The largest FC overlap, 0.83 , occurs in the $\mathrm{I}\left(\Omega= \pm \frac{3}{2}\right)$ state. For the III $\left(\Omega= \pm \frac{1}{2}\right)$ state, considerable overlaps are observed between the vibrational states with lower quantum numbers. The overlap of the two ground vibrational levels is 0.75 , which occurs because the $\operatorname{III}\left(\Omega= \pm \frac{1}{2}\right)$ and $\mathrm{I}\left(\Omega= \pm \frac{1}{2}\right)$ states have similar equilibrium positions. As for the $\operatorname{II}\left(\Omega= \pm \frac{1}{2}\right)$ and $\mathrm{I}\left(\Omega= \pm \frac{3}{2}\right)$ states, their equilibrium positions are $1 a_{0}$ shorter than that of the ground state equilibrium position, and the $\mathrm{FC}$ overlaps between the lower vibrational states are about $\sim 0.1$ or smaller. There is a clear bend in the pattern of the strong overlap for the II $\left(\Omega= \pm \frac{1}{2}\right)$ state after $\nu_{g}=15$ and $\nu_{e}=45$. It is consistent with the corresponding potentials, where there is an avoided crossing between the two $\Omega= \pm \frac{1}{2}$ states. A similar pattern is seen in the $\operatorname{III}\left(\Omega= \pm \frac{1}{2}\right)$ state FC overlaps.

\section{H. Dipole moment and static dipole polarizability}

The dipole moment $\mu$ and dipole polarizability $\alpha$ are sensitive to electron correlation and the choice of basis functions. We have shown ${ }^{16}$ that the dipole polarizability of the $\mathrm{Yb}$ atom can be accurately predicted at the CC level of theory with DKH3 and RECP approximations. The same ANO-RCC basis for DKH3 and valence basis with RECP approaches were used. We computed $\mu$ and $\alpha$ by means of the FF procedure for the $\mathrm{LiYb}$ molecule using the $\mathrm{UCCSD}(\mathrm{T})$ method with the DKH3 and RECP approaches. The vibrationally averaged results $\mu_{v}$ and $\alpha_{v}$ are given in 


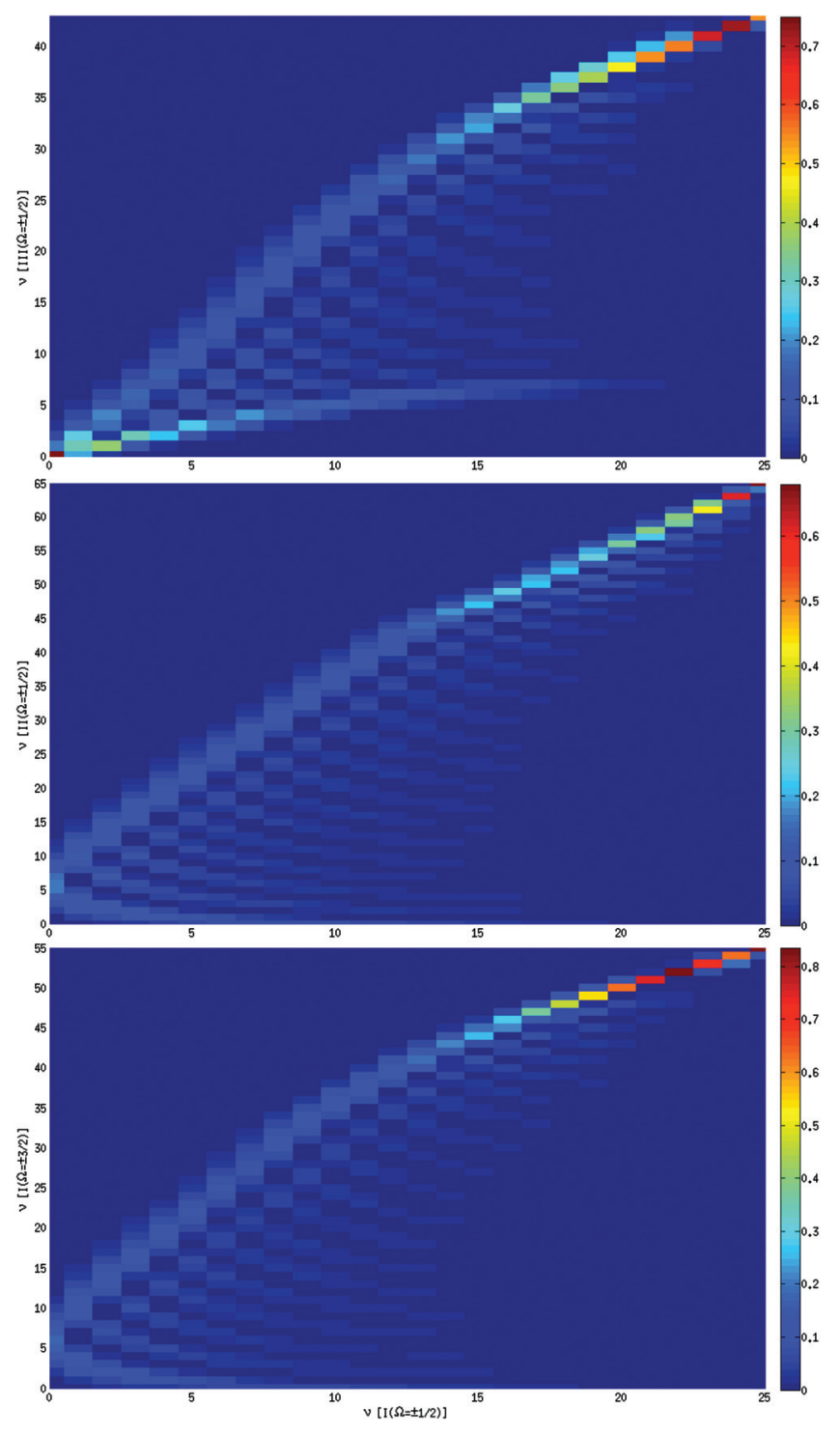

FIG. 4. Franck-Condon factors as functions of the vibrational quantum numbers of the ground (horizontal axis) and excited states (vertical axis).

Tables VI and VII along with the corresponding values $\mu_{e}$ and $\alpha_{e}$ obtained at the equilibrium separation. The variations of these quantities as a function of $R$ are depicted in Fig. 5. For comparison, the dipole moment derived as the expectation value with the SS-MRCI wave functions are presented in Table VI and Fig. 5. The sign convention is that the dipole moment is positive if the polarity is $\mathrm{Li}^{-} \mathrm{Yb}^{+}$.

TABLE VI. The ground state dipole moments at $R_{e}\left(\mu_{e}\right)$ and the vibrationally averaged dipole moments $\left(\mu_{v}\right)$ computed at the coupled-cluster and multireference configuration interaction levels of theory. The sign convention is that the dipole moment of $\mathrm{LiYb}$ is considered positive if the polarity is $\mathrm{Li}^{-} \mathrm{Yb}^{+}$

\begin{tabular}{lccr}
\hline \hline & & \multicolumn{1}{c}{$\mu_{e}$} & \multicolumn{1}{c}{$\mu_{v}$} \\
Theory & Method & (D) & \multicolumn{1}{c}{ (D) } \\
\hline RECP-UCCSD(T) & FF & -0.058 & -0.058 \\
DKH3-UCCSD(T) & FF & -0.028 & -0.030 \\
SS-MRCISD(Q) & FF & 0.135 & 0.128 \\
SS-MRCISD & FF & 0.095 & 0.085 \\
SS-MRCISD & EV & -0.114 & -0.105 \\
\hline \hline
\end{tabular}

TABLE VII. The ground state dipole polarizability $\alpha_{e}$ (a.u.) at $R_{e}$ and the vibrationally averaged dipole polarizability $\alpha_{v}(v=0)$ computed at the UCCSD(T) level of theory. The subscripts $\|$ and $\perp$ label the parallel and perpendicular components of the polarizability.

\begin{tabular}{lcccc}
\hline \hline & $\alpha_{\|}^{e}$ & $\alpha_{\|}^{v}$ & $\alpha_{\perp}^{e}$ & $\alpha_{\perp}^{v}$ \\
\hline RECP-UCCSD(T) & 584 & $\ldots$ & 235 & $\ldots$ \\
DKH3-UCCSD(T) & 585 & 584 & 233 & 233 \\
\hline \hline
\end{tabular}

There are large differences in $\mu$ among various theoretical treatments. However, the computed $\mu$ shows a similar behavior as a function of $R$. At large $R, \mu$ is small and negative. As $R$ decreases, the magnitude of $\mu$ increases and reaches a maximum. Further reducing $R, \mu$ starts to decrease and changes sign. At small $R, \mu$ is positive and large with small oscillations near the equilibrium distance. The variation of $\mu$ as a function $R$ reflects the electron distribution in the molecule and may be understood qualitatively by the atomic electronegativity (EN), which measures the ability of the atom to attract electrons when combined with other atoms and is related to fundamental atomic properties including the ionization potential (IP) and the electron affinity (EA). $\mathrm{IP}_{\mathrm{Li}}=5.3917 \mathrm{eV}, \mathrm{IP}_{\mathrm{Yb}}=6.2542 \mathrm{eV}, \mathrm{EA}_{\mathrm{Li}}=0.6173 \mathrm{eV}$, and $\mathrm{EA}_{\mathrm{Yb}}=0.518 \mathrm{eV}$. The $\mathrm{EN}$ of $\mathrm{Li}$ and $\mathrm{Yb}$ atom are close to each other in any of various definitions. ${ }^{45}$ The ENs of $\mathrm{Yb}$ and $\mathrm{Li}$ are, respectively, 1.26 and 0.98 . $^{46}$ Therefore, at large $R$, the net charge transfer from $\mathrm{Li}$ to $\mathrm{Yb}$ is small and $\mathrm{LiYb}$ has a small negative $\mu$. As the two atoms approach each other, the electron density close to $\mathrm{Yb}$ increases and the magnitude of $\mu$ increases. When they are close enough to start to form a weak $\sigma$ bond, the electron density moves toward the $\mathrm{Li}$ atom as the $\sigma$ bonding occurs. As a result, the magnitude of $\mu$ starts to decrease. As the atoms further approach, the weight of excited state configurations in the ground state wave function increases, resulting in a large variation of the dipole moment.

The expectation formalism (EV) overestimates $\mu$ at short $R$, as demonstrated by the FF results. At large $R, \mathrm{EV}$ gets the sign wrong. The improved agreement with the $\mathrm{UCCSD}(\mathrm{T})$ values from the FF MRCISD to $\operatorname{MRCISD}(\mathrm{Q})$ calculations shows the importance of higher order excitations in the description of the dynamic correlation, as also revealed by the potential depths computed by the corresponding theory. The two CCSD $(\mathrm{T})$ results differ by less than $10 \%$ for $R>8 a_{0}$. However, the deviation is significant around the equilibrium distance. The small changes in the potential depth due to the CP corrections suggest that the large difference in $\mu$ does not result from BSSE. The CP corrected dipole moment calculated at the DKH3-CCSD(T) level of theory and plotted in Fig. 4 differs by less than $6 \%$, relative to the one without the CP corrections. The large difference between the DKH3 and RECP results is likely a consequence of the basis set effects.

Small dipole moments $\mu_{e}=-0.028 \mathrm{D}$ (Debye) and $\mu_{v}$ $=-0.030 \mathrm{D}$ are predicted by the $\mathrm{CP}$ corrected DKH3$\mathrm{UCCSD}(\mathrm{T})$ approach. The RECP approximation yields a larger value, $-0.058 \mathrm{D}$ for both $\mu_{e}$ and $\mu_{v}$. In both cases, the magnitude of the dipole moment is small. The large dipole 


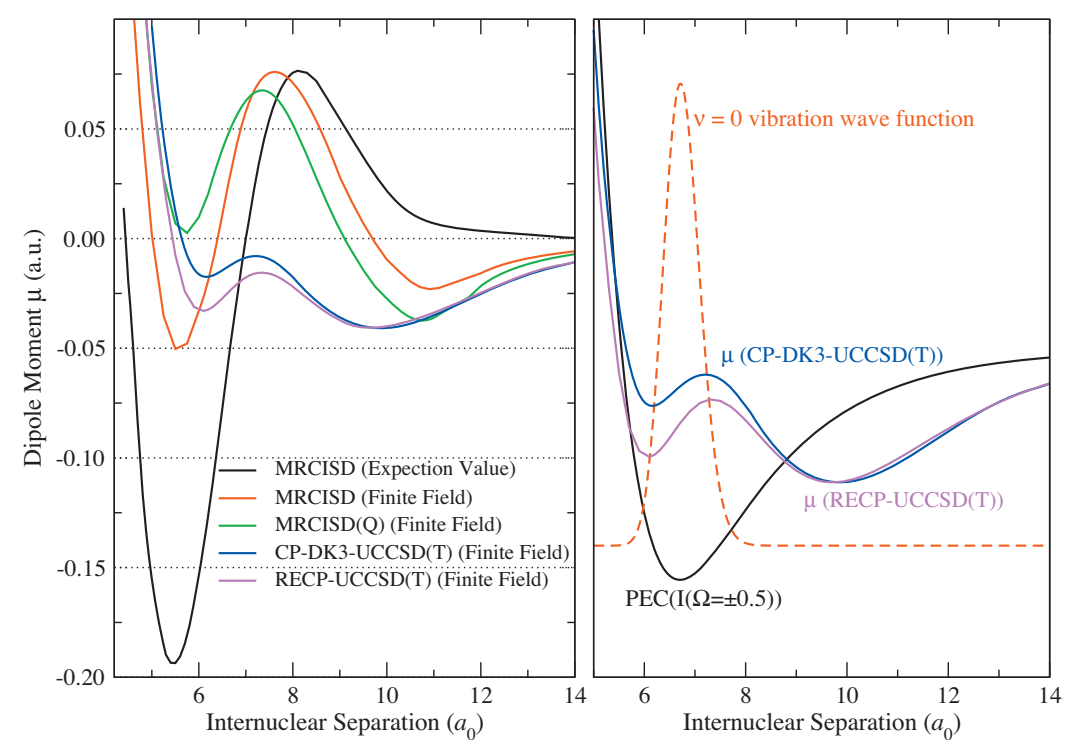

FIG. 5. The ground state dipole moment of the LiYb molecule as a function of the internuclear distance $R$ computed at several levels of theory (left panel). A schematic representation of the ground state potential energy curve, the wave function of the ground vibrational state, and the computed dipole moments (right panel). moment at short $R$ has little contribution because the vibrational wave function is localized in the equilibrium region. A schematic representation of the corresponding vibrational wave function (for $\nu=0$ ), the ground state PEC, and dipole moment functions is depicted in Fig. 5. In a recent calculation of $\mathrm{RbYb},{ }^{42}$ a dipole moment of $\sim 0.98 \mathrm{D}$ was derived from the FF calculations at the CCSD(T) level of theory with a SF Hamiltonian of Dyall. ${ }^{47}$ We tested our methods by calculating the dipole moment of RbYb with the RECP approach at the $\operatorname{CCSD}(\mathrm{T})$ level of theory (correlating the same 23 electrons as in Ref. 42) and obtained a value of $0.88 \mathrm{D}$ at the equilibrium distance, consistent with the result in Ref. 42.

The dipole polarizabilities derived from these two $\operatorname{CCSD}(\mathrm{T})$ calculations are close to each other. The results are summarized in Table VII. The parallel components $\alpha_{\|}^{e}$ at the equilibrium distances are 585 and 584 a.u. for the DK3 and RECP approaches, respectively. Similar agreement is found for other internuclear distances, and the deviation is less than $\sim 1 \%$. The vibrationally averaged value $\alpha_{\|}^{v}$ from the DK3 approach is 584 a.u. The corresponding perpendicular components $\alpha_{\perp}^{e}$ and $\alpha_{\perp}^{v}$ are all 233 a.u. At $R=18.0 a_{0}$, our calculated $\alpha_{\|}=326$ a.u. and $\alpha_{\perp}=301$ a.u. are consistent with the sum of the atomic values $\alpha_{\mathrm{Li}+\mathrm{Yb}}=306$ a.u., with $\alpha_{\mathrm{Yb}}$ $=142$ a.u. (Ref. 16) and $\alpha_{\mathrm{Li}}=164$ a.u. (Ref. 35).

\section{SUMMARY AND CONCLUSION}

We systematically examine the PECs of the ground and low-lying excited states of the LiYb molecule using coupledcluster and multireference configuration interaction methods. The scalar relativistic effects are successfully included by both the relativistic pseudopotential approach and the thirdorder Douglas-Kroll-Hess Hamiltonian. The spin-orbit interaction is taken into account by means of the full Breit-Pauli Hamiltonian at the MRCISD level of theory and the full spin-orbit states are obtained perturbatively by the state interacting method. We also derive the long-range dispersion coefficients for the ground and first two excited states. We further report the spectroscopic constants and Frank-Condon overlaps between the ground and excited states.
Compared to the $\mathrm{CC}$ theory, the MRCI method using the state-averaged CASSCF reference wave function substantially underestimates the potential depth and long-range interaction. When replaced by the state-specific CASSCF reference wave function, improved agreement is observed, but the potential depth is still undervalued by about $10 \%$. The counterpoise corrections of the BSSE at the $\operatorname{CCSD}(\mathrm{T})$ level of theory decreases the potential depth by about $10 \%$. Therefore, a cancellation of error leads to the close agreement between CP-CCSD(T) and the SS-MRCI without CP correction.

The first three excited states associated with the $\operatorname{Li}\left({ }^{2} \mathrm{P}\right)$ $+\mathrm{Yb}\left({ }^{1} \mathrm{~S}\right)$ manifold were carefully examined by means of the SS-MRCI method. Large Franck-Condon overlaps with the ground state are observed, which could be possible routes for the production of cold $\mathrm{LiYb}$ molecule through the twophoton assisted photoassociation. The nature of the higher excited states is complicated due to the interactions with the states from the $\mathrm{Yb}(4 f-5 d)$ transition, where a more rigorous relativistic treatment is necessary but difficult to compute.

The CC calculations predict a permanent dipole moment of 0.03-0.06 D for the ground state of LiYb. Compared to other polar molecules, such as $\mathrm{RbYb}(0.98 \mathrm{D}){ }^{42} \mathrm{LiCs}$ $(5.5 \mathrm{D}),{ }^{48} \mathrm{RbCs}(1.25 \mathrm{D}),{ }^{49}$ and $\mathrm{KRb}(0.76 \mathrm{D}),{ }^{50}$ the LiYb dipole moment is small.

It is interesting to infer the tendency of the diatomic molecules composed of $\mathrm{Yb}$ and alkali-metal atoms from the known molecular data. The diatomic interaction potentials between the He atom and alkali-metal atoms can be grouped into two sets. ${ }^{51}$ LiHe and $\mathrm{NaHe}$ have a similar interaction strength, as do KHe and RbHe. The atomic polarizabilities ${ }^{52}$ of the alkali-metal atoms share the same tendency, $\alpha_{\mathrm{Li}}$ $\approx \alpha_{\mathrm{Na}}$ and $\alpha_{\mathrm{K}} \approx \alpha_{\mathrm{Rb}}$. For $\mathrm{RbYb},{ }^{42} D_{e}=804 \mathrm{~cm}^{-1}$ and $\mu_{v}$ $=0.98 \mathrm{D}$. $\mathrm{Yb}$ resembles the He atom. It is likely that $\mathrm{NaYb}$ has a similar $D_{e}$ and $\mu_{v}$ to $\mathrm{LiYb}$, whereas the properties of $\mathrm{KYb}$ lie closer to those of RbYb. The dipole polarizability of the Cs atom is larger than $\mathrm{Rb}$ by about 90 a.u. and the dipole moment of $\mathrm{CsYb}$ could be larger than that of $\mathrm{RbYb}$. 


\section{ACKNOWLEDGMENTS}

The research of A.D. and P.Z. is supported by the Chemical Science, Geoscience, and Bioscience Division of the Office of Basic Energy Science, Office of Science, U.S. Department of Energy, and of H.R.S. by a NSF grant to ITAMP. We thank Y. Takahashi for helpful discussions and Z.-C. Yan for providing the $\mathrm{Li}$ atomic spectrum data. NSF Teragrid Grant No. TG-Phy 090079 was used for the computations.

${ }^{1}$ L. D. Carr, D. DeMille, R. V. Krems, and J. Ye, New J. Phys. 11, 055049 (2009).

${ }^{2}$ R. V. Krems, B. Friedrich, and W. C. Stwalley, Cold Molecules: Theory, Experiment, Applications (CRC, Boca Raton, 2009).

${ }^{3}$ M. Greiner, C. A. Regal, and D. S. Jin, Nature (London) 426, 537 (2003).

${ }^{4}$ K.-K. Ni, S. Ospelkaus, M. H. G. de Miranda, A. Pe'er, B. Neyenhuis, J. J. Zirbel, S. Kotochigova, P. S. Julienne, D. S. Jin, and J. Ye, Science 322, 231 (2008).

${ }^{5}$ J. M. Sage, S. Sainis, T. Bergeman, and D. DeMille, Phys. Rev. Lett. 94, 203001 (2005).

${ }^{6}$ M. L. Wall and L. D. Carr, New J. Phys. 11, 055027 (2009).

${ }^{7}$ A. Micheli, G. K. Brennen, and P. Zoller, Nat. Phys. 2, 341 (2006).

${ }^{8}$ J. Doyle, B. Friedrich, R. V. Krems, and F. Masnou-Seeuws, Eur. Phys. J. D 31, 49 (2001); R. V. Krems, Phys. Chem. Chem. Phys. 10, 4079 (2008)

${ }^{9}$ J. D. Weinstein, R. deCarvalho, T. Guillet, B. Friedrich, and J. M. Doyle, Nature (London) 395, 148 (1998).

${ }^{10}$ T. Köhler, K. Góral, and P. S. Julienne, Rev. Mod. Phys. 78, 1311 (2006)

${ }^{11}$ K. M. Jones, E. Tiesinga, P. D. Lett, and P. S. Julienne, Rev. Mod. Phys. 78, 483 (2006).

${ }^{12}$ N. Nemitz, F. Baumer, F. Münchow, S. Tassy, and A. Görlitz, Phys. Rev. A 79, 061403(R) (2009).

${ }^{13}$ Y. Takasu and Y. Takahashi, J. Phys. Soc. Jpn. 78, 012001 (2009).

${ }^{14}$ B. Marcelis, S. J. J. M. F. Kokkelmans, G. V. Shlyapnikov, and D. S. Petrov, Phys. Rev. A 77, 032707 (2008).

${ }^{15}$ M. Okano, H. Hara, M. Muramatsu, K. Doi, S. Uetake, Y. Takasu, and Y. Takahashi, Appl. Phys. B 98, 691 (2010).

${ }^{16}$ P. Zhang and A. Dalgarno, J. Phys. Chem. A 111, 12471 (2007); Mol. Phys. 106, 1525 (2008)

${ }^{17}$ M. Douglas and N. M. Kroll, Ann. Phys. (N.Y.) 82, 89 (1974); A. Wolf, M. Reiher, and B. A. Hess, J. Chem. Phys. 117, 9215 (2002).

${ }^{18}$ Y. Wang and M. Dolg, Theor. Chem. Acc. 100, 124 (1998).

${ }^{19}$ B. K. Sahoo and B. P. Das, Phys. Rev. A 77, 062516 (2008).

${ }^{20}$ Y. Ralchenko, A. E. Kramida, J. Reader, and NIST ASD Team, NIST Atomic Spectra Database, version 3.1.5 (2008) (Online). Available at http://physics.nist.gov/asd3, National Institute of Standards and Technology, Gaithersburg, MD (29 September 2009).

${ }^{21}$ A. Berning, M. Schweizer, H.-J. Werner, P. J. Knowles, and P. Palmieri,
Mol. Phys. 98, 1823 (2000).

${ }^{22}$ P. J. Knowles, C. Hampel, and H.-J. Werner, J. Chem. Phys. 99, 5219 (1993).

${ }^{23}$ J. D. Watts, J. Gauss, and R. J. Bartlett, J. Chem. Phys. 98, 8718 (1993).

${ }^{24}$ H.-J. Werner and P. J. Knowles, J. Chem. Phys. 89, 5803 (1988); Chem. Phys. Lett. 145, 514 (1988).

${ }^{25}$ S. R. Langhoff and E. R. Davidson, Int. J. Quantum Chem. 8, 61 (1974).

${ }^{26}$ H.-J. Werner and P. J. Knowles, J. Chem. Phys. 82, 5053 (1985); Chem. Phys. Lett. 115, 259 (1985).

${ }^{27}$ MOLPRO, a package of ab initio programs written by H.-J. Werner, P. J. Knowles, R. Lindh et al.

${ }^{28}$ K. A. Peterson, personal communication (2009).

${ }^{29}$ B. O. Roos, R. Lindal, P.-A. Malmqvist, V. Veryazov, and P.-O. Widmark, J. Phys. Chem. A 112, 11431 (2008).

${ }^{30}$ W. A. de Jong, R. J. Harrison, and D. A. Dixon, J. Chem. Phys. 114, 48 (2001).

${ }^{31}$ F. B. van Duijneveldt, J. G. C. M. van Duijneveldt-van de Rijdt, and J. H. van Lenthe, Chem. Rev. (Washington, D.C.) 94, 1873 (1994); G. Chałasiński and M. M. Szczęńniak, ibid. 100, 4227 (2000).

${ }^{32}$ S. F. Boys and F. Bernardi, Mol. Phys. 19, 553 (1970).

${ }^{33}$ R. J. Vos, F. B. van Duijneveldt, and J. H. van Lenthe, Mol. Phys. 67, 1011 (1989)

${ }^{34}$ D. Tzeli and A. Mavridis, J. Chem. Phys. 118, 4984 (2003).

${ }^{35}$ L.-Y. Tang, Z.-C. Yan, T.-Y. Shi, and J. F. Babb, Phys. Rev. A 79, 062712 (2009).

${ }^{36}$ C. Zhu, A. Dalgarno, S. G. Porsev, and A. Derevianko, Phys. Rev. A 70, 032722 (2004).

${ }^{37}$ M. Marinescu and H. R. Sadeghpour, Phys. Rev. A 59, 390 (1999); J.-Y. Zhang and J. Mitroy, ibid. 76, 022705 (2007).

${ }^{38}$ DALTON, a molecular electronic structure program, Release 2.0 (2005). See http://daltonprogram.org.

${ }^{39}$ C. Adamo and V. Barone, J. Chem. Phys. 110, 6158 (1999).

${ }^{40}$ C. C. Marston and G. G. Balint-Kurti, J. Chem. Phys. 91, 3571 (1989).

${ }^{41}$ A. Tkatchenko and M. Scheffler, Phys. Rev. Lett. 102, 073005 (2009).

${ }^{42}$ L. K. Sørensen, S. Kencht, T. Fleig, and C. M. Marian, J. Phys. Chem. A 113, 12607 (2009).

${ }^{43}$ T. J. Lee and P. R. Taylor, Int. J. Quantum Chem. S23, 199 (1989).

${ }^{44}$ J. M. Hutson, Comput. Phys. Commun. 84, 1 (1994).

${ }^{45}$ N. C. Jeong, J. S. Lee, E. L. Tae, Y. J. Lee, and K. B. Yoon, Angew. Chem., Int. Ed. 47, 10128 (2008).

${ }^{46}$ L. Pauling, J. Am. Chem. Soc. 54, 3570 (1932).

${ }^{47}$ K. G. Dyall, J. Chem. Phys. 100, 2118 (1994).

${ }^{48}$ M. Aymar and O. Dulieu, J. Chem. Phys. 122, 204302 (2005).

${ }^{49}$ S. Kotochigova and E. Tiesinga, J. Chem. Phys. 123, 174304 (2005).

${ }^{50}$ S. Kotochigova, P. S. Julienne, and E. Tiesinga, Phys. Rev. A 68, 022501 (2003).

${ }^{51}$ T. V. Tscherbul, P. Zhang, H. R. Sadeghpour, and A. Dalgarno, Phys. Rev. A 79, 062707 (2009).

${ }^{52}$ A. Derevianko, J. F. Babb, and A. Dalgarno, Phys. Rev. A 63, 052704 (2001); A. Derevianko, S. G. Porsev, and J. F. Babb, At. Data Nucl. Data Tables 96, 323 (2010). 